\title{
Soft Robotic Hand Exoskeleton Systems: Review and Challenges Surrounding the Technology
}

\author{
Qiaoling Meng*, Shichuan Xiang and Hongliu Yu \\ Institute of rehabilitation engineering and technology, University of Shanghai for Science and Technology, Shanghai, P. R. China \\ Shanghai Engineering Research Center of Assistive Devices, Shanghai, P. R. China \\ Key Laboratory of Neural-functional Information and Rehabilitation Engineering of the Ministry of Civil Affairs, Shanghai, \\ P. R .China \\ ${ }^{*}$ Corresponding author
}

\begin{abstract}
Soft robotic hand exoskeleton systems are taken into account to solve the problems happened in the hand rehabilitation, such as long-term training for stroke patients and heavy workload for physicians. These systems are one of the hot issues in recent robotic research because that they are not only applied in rehabilitation but also in assisting the human beings. With the difference of the rigid robotic exoskeleton hand systems, the soft exoskeleton hand systems should be designed together with the human hand since they directly interact with the patients. This paper briefly reviews different robotic hand exoskeleton systems from two aspects: mechanical design and control system. The existing soft hand exoskeleton technologies and challenges are discussed in this paper.
\end{abstract}

Keywords-exoskeleton; rehabilitation; hand rehabilitation; wearalbe robot; hand exoskeleton; soft robot; soft glove; powerassist; rehabilitation robotics; flexure hinge

\section{INTRODUCTION}

A robotic hand exoskeleton system (or an exoskeleton hand robot) is not a new technology, in which it has been focused by researchers since 1960s [1, 2]. It fully merges the human intelligence and machine power. This type of systems is a wearable intelligent glove (a wearable robot hand) intended for augmenting or enhancing the abilities of human hands. At the first, these robot systems are researched mainly for military in order to augment the human power [2]. With the development of robotics in medical, the rehabilitation purpose is given to the robotic hand exoskeleton intended to assist the physically impaired patients to do their training [[3]]. However, there are lots of patients' hands cannot recover their abilities. Therefore, researchers start to take into account the material and geometrical flexibilities to assist the patients with impaired hands to do the tasks in the life.

In the application of hand exoskeletons for hand rehabilitation, its aim to recover and restore the hand movements of patients. The hand disabilities is caused mainly by the cerebral vascular deceases (stroke)[ [4]]. For such an application, patients can train their hands immediately after their surgeries. The rehabilitation cycle is still long for the patients because that they only do the exercise for 20-30 minutes every day. On the other hand, therefore, the ability of assisting the human to perform basic activities of daily living (ADL) is also needed to be considered in the robotic hand exoskeleton system researches [[5]]. Because of such the inherent motor restored and assisted requirements, soft robotic hand exoskeleton systems are increasingly developed recently. These requirements have inspired considerable developments in robotic hands in terms of their size, quality and degree of freedom (DOF), flexibilities, lightness and dexterous manipulation capabilities. Hence, the choice of actuator, the finger exoskeleton mechanical design, the choice of DOF, the usage of the desired robotic hand exoskeleton system, and the control of this kind of intelligent system should be taken into account during the design or research of a soft robotic hand exoskeleton system.

This paper briefly reviews the robotic hand exoskeleton systems for rehabilitation. The review work were proceeding by searching the main databases such as PubMed, the Institute of Electrical and Electronics Engineers (IEEE), Science Direct with the key words exoskeleton; rehabilitation; hand rehabilitation; wearable robot; hand exoskeleton; soft robot; soft glove; power-assist; rehabilitation robotics; flexure hinge. The common problems in design of a soft robotic hand exoskeleton system such as actuator problems, the DOF definition, and the mechanical design are discussed in these following sections.

\section{DeSign OF A Soft Robotic HAND EXOSKELETON SYSTEM}

The design of a soft robotic hand exoskeleton is a high complexity task due to various requirements and issues which exist in the meantime. As in [[6]], Mohamamd Mozaffari Foumashi et al. have done a review work on the robotic hand exoskeleton systems in 2011. Although most of the work described in the report is about the rigid robotic hand exoskeleton system, they summarized and discussed the problems in the design of a robotic hand exoskeleton system. These problems can be classified into two parts: one is the mechanical design, and the other one is control scheme. In the mechanical design, the number of DOFs of hand exoskeleton, the number of actuators, system architecture, and transmission system should be taken into account. As for the control scheme, the problems of the control signals, sensors, encoders, and the position of these sensors should be considered by the designers. Besides that, there are other problems of equal importance in the design of soft robotic hand exoskeleton system. For 
instance, the flexible segments used to active the fingers is quite important problem for the designers. The following contents describe the soft robotic hand exoskeleton system from mechanical design and control scheme after reviewing13 soft robotic hand exoskeleton systems (summarized in Table I).

TABLE I. SOFT ROBOTIC HAND EXOSKELETON SYSTEMS

\begin{tabular}{|c|c|c|c|c|c|}
\hline No. & Name & $\begin{array}{c}\text { Prime } \\
\text { mover } \\
\text { types }\end{array}$ & DOFs & Flexible deformation unit & Function \\
\hline 1 & OHAE $E^{[[7]]}$ & Motor & 3 & Flexible rod and rope drive & Rehabilitation and daily life-assisted \\
\hline 2 & SNU Exo-Glove $e^{[[8]]}$ & Motor & 1 & Flexible rod and rope drive & Rehabilitation and daily life-assisted \\
\hline 3 & The SEM Glove $\mathrm{e}^{[[9]]}$ & Motor & 3 & Elastomer and rope drive & Rehabilitation and daily life-assisted \\
\hline 4 & Worcester Polytechnic Institute [[10]] & Motor & 5 & Elastomer and rope drive & Rehabilitation training \\
\hline 5 & University of Strathclyde [[11]] & Motor & 3 & Elastomer and rope drive & Rehabilitation training \\
\hline 6 & $\begin{array}{lll}\begin{array}{l}\text { The National } \\
\text { Okayama I[12]] }\end{array} & \text { University } & \text { of } \\
\end{array}$ & Air pump & 6 & Elastomer and pneumatic drive & Rehabilitation and daily life-assisted \\
\hline 7 & $\begin{array}{lcc}\begin{array}{l}\text { The National } \\
\text { Okayama II[[13]] }\end{array} & \text { University } & \text { of } \\
\end{array}$ & Air pump & 6 & Elastomer and pneumatic drive & Rehabilitation and daily life-assisted \\
\hline 8 & Japan's Toshiba Corp ${ }^{[[14]]}$ & Air pump & 4 & Elastomer and pneumatic drive & Rehabilitation and daily life-assisted \\
\hline 9 & $\begin{array}{l}\text { National University of Singapore } \\
\text { [[15]] }^{[15}\end{array}$ & Air pump & 1 & Elastomer and pneumatic drive & Rehabilitation and daily life-assisted \\
\hline 10 & MRC-Glove ${ }^{[[16]]}$ & Air pump & 4 & Elastomer and pneumatic drive & Rehabilitation training \\
\hline 11 & $\begin{array}{l}\text { National University of Singapore } \\
\text { II }^{[[17]]}\end{array}$ & Air pump & 3 & Elastomer and pneumatic drive & Rehabilitation training \\
\hline 12 & Harvard University I[ [18], [3] ] & $\begin{array}{l}\text { hydraulic } \\
\text { pump }\end{array}$ & 5 & Elastomer and hydraulic drive & Rehabilitation training \\
\hline 13 & Harvard University II[[19]] & $\begin{array}{l}\text { hydraulic } \\
\text { pump }\end{array}$ & 5 & Elastomer and hydraulic drive & Rehabilitation and daily life-assisted \\
\hline
\end{tabular}

\section{A. Mechanical Design}

As what we known about the mechanical design, different design criteria should be considered, especially in the design of soft exoskeleton. Onen et al. [[20]] addressed the design criteria for the lower extremity exoskeletons. The criteria are also good for the design of hand exoskeletons, they are ergonomic and comfortable design, high maneuverability, lightweight and strong structure, and adaptability to different users, and user safety. These design criteria are the key to define the numbers of DOFs and actuators, to design the flexible segments for moving the fingers in terms of the design of a hand exoskeleton.

\section{1) $\mathrm{DOFs}$}

In Table I, different soft robotic hand exoskeleton systems present a different number of DOFs due to their design requirements (or the characteristics of the desired hand exoskeletons). A hand has 20 DOFs [[21]], they are the flexion/extension DOF at each interphalangeal (IP) and each metatarsophalangeal (MCP) as well as both flexion/extension and abduction/adduction DOFs at the Carpometacarpal (CMC). In general, a designer defines the number of DOFs in order to imitate the movements of hand. However, there are no systems that can implement 20 DOFs even though they adopted underactuated mechanisms. The main two reasons why the20 DOFs cannot be realized in a soft hand exoskeleton are that the coupled DOFs of MCP and CMC and the numbers of actuators. It is worth to study in further coupled mechanism with multi DOFs.

\section{2) Actuators}

According to what mentioned above, the number of the actuators is a key design parameter in soft hand exoskeletons. This problem can be discussed by design an under-actuated mechanism. However, there is another important problem in the design of the system. That is the choice of actuators. There are pneumatic, hydraulic, electrical, piezoelectric, electro active polymers actuators that can be used in robotic applications. In particular, the pneumatic, hydraulic and electrical actuators are the common actuators in soft robotic hand exoskeleton systems (as shown in Table 1.) because that large moment and displacement are needed. However, the pneumatic and hydraulic actuators have some disadvantages such as the heavy attached and small output moment (for instance, Harvard University Hand shown in Figure 2 (e)). Therefore, large output moment, large route actuators should be developed in the future in order to meet the criteria of lightweight and high maneuverability.

\section{3) Flexible deformation units}

According to the criteria, a soft robotic hand exoskeleton system needs to consider the wearer's feeling about wearing and controllable. Therefore, the choice of material and design of the active soft mechanism for per finger or whole hand are very important. OHAE [[7]](shown in Figure 1a) adopted 0.020 ' diameter carbon fiber rods to produce the movement of the phalanges and utilized a 0.014', diameter braided polymer cable in a pull-pull segment in order to provide the moments at each joint. The flexibility of the mechanism plays the key role in the ergonomic and comfortable of this type of systems. This type of flexible rods is a flexure hinge used to 
substitute the rigid revolute joint in compliant mechanisms. However, the motion range can be limited by the material and geometrical properties and the deformation of flexible rod can affect the comfortable. SNU Exo-Glove[[8]] (as shown in Figure 2b) simplified the shape of flexible rod of each finger in order to promote the comfort and controllable.

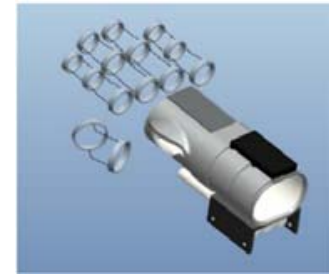

(a) OHAE

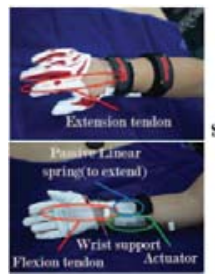

(b) SNU Exo-Glove
FIGURE I. TWO TYPICAL SOFT ROBOTIC HAND EXOSKELETON SYSTEMS WITH FLEXIBLE RODS

Recently, the elastomer with the linear actuators such as pneumatic is attracted lots of researchers. Most of this type of robotic hand exoskeleton systems' deformation units (the segments are utilized to active the fingers) were designed together with properties of material and geometry. Such design can meet lightweight For instance, The National University of Okayama I[[12]] (as shown in Figure 2(a), The National University of Okayama II[[13]] (as shown in Figure 2(b), National University of Singapore I[[15]] (as shown in Figure 2(c), MRC-Glove[[16]] (as shown in Figure 2(d) , Harvard University I[[3, 19]] (as shown in Figure 2(e).

\section{B. Control System}

The control systems reviewed in this paper are listed in Table 2.

\section{1) Control scheme}

There are two main control strategies in robotic system for rehabilitation that are "high-" and "low- level" control strategies [[5]]. "High-level” control strategy is that the motor plasticity will be driven explicitly. For example, assistive control algorithm, challenge-based control algorithm. This type of control algorithms can promote the intelligence of the soft robotic hand exoskeleton systems. Unfortunately, there are a few researches present this control algorithms due to their complexity. The force, position, impedance or admittance factors of the "high-level" control strategies can be control objects in "Low-level" control strategies. Actually, most of "high-level" control strategies combine some "low-level" control algorithms such as the Harvard University I (as shown in Table II.). The EMG signals are responsible for the activation and deactivation of a low-level controller.

\section{2) Control signals}

In Table II, kinematic, dynamic or surface electromyography (EMG) is the common control signals in soft robotic hand exoskeleton systems. The kinematic and dynamic signals are used as control input switches in a lowlevel control strategies. For instance, OHAE used the force sensing resistors and potentiometer to transduce the finger movement effort to the controller as feedback signals [[7]].In general, a wearer's intentions can be captured by EMG signals. Therefore, EMG signals are often used as the switch signal in low-level strategies in order to develop it control algorithm into high-level control strategies. For instance, Harvard University Hand I and II [[3, 18, 19]], they adopted the EMG signals as the control switch signal to implement the human intention control scheme. The National University of Okayama also uses the EMG signal to realize their intelligent control system.
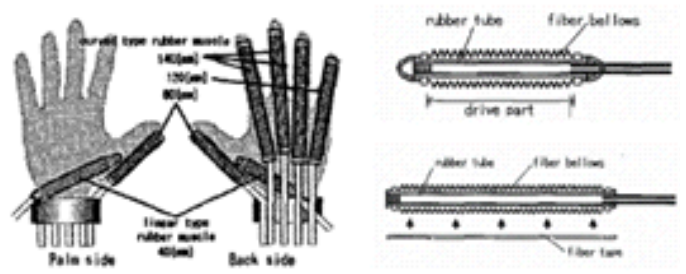

(a) Soft glove and pneumatic muscles of The National University of Okayama

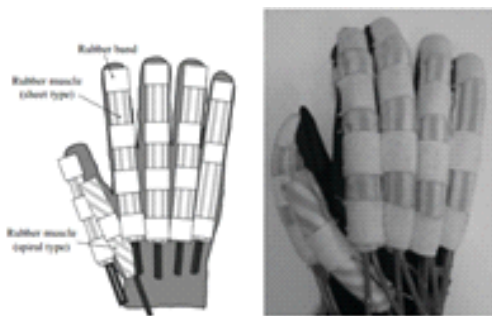

(b) Soft glove of The National University of Okayama

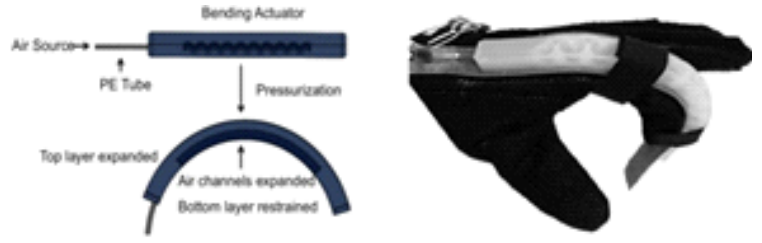

(c) National University of Singapore I

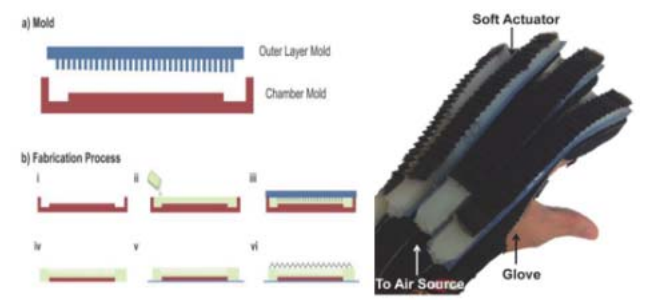

(d) MRC-Glove

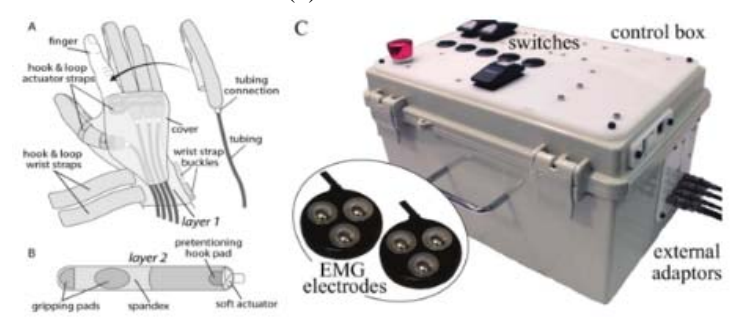

(e) Harvard University I

FIGURE II. FIVE TYPICIAL TYPE OF SOFT ROBOTIC HAND EXOSKELETON SYSTEM WITH ELASTOMER 
TABLE II. CONTROL SIGNALS AND STRATEGIES OF COMPLIANT EXOSKELETON REHABILITATION ROBOTIC HANDS

\begin{tabular}{|c|c|c|c|}
\hline Name & Driving finger & Control signals & Control strategies \\
\hline $\mathrm{OHAE}^{[[7]]}$ & Thumb, index finger, middle finger & Dynamic signals & "Low-level” control strategy \\
\hline SNU Exo-Glove $e^{[[8]]}$ & Thumb, index finger, middle finger & EMG signal & $\begin{array}{c}\text { Challenge-based and EMG-based "Low-level" } \\
\text { control strategy }\end{array}$ \\
\hline The SEM Glove ${ }^{[[9]]}$ & Thumb, middle finger, ring finger & Dynamic signals & EMG-based "Low-level” control strategy y \\
\hline $\begin{array}{l}\text { Worcester Polytechnic Institute } \\
{[[10]]}\end{array}$ & All fingers & $\begin{array}{l}\text { EMG signal and kinematics } \\
\text { signals } \\
\end{array}$ & Impedance control strategy \\
\hline University of Strathclyde [[11]] & Thumb, index finger, middle finger & EMG signal & EMG-based “Low-level” control strategy \\
\hline $\begin{array}{c}\text { The National University of } \\
\text { Okayama I[122]] }\end{array}$ & All fingers & EMG signal & Performance-based adaptive control strategy \\
\hline $\begin{array}{c}\text { The National University of } \\
\text { Okayama II }{ }^{[13]]}\end{array}$ & All fingers & EMG signal & EMG-based "Low-level” control strategy y \\
\hline Japan's Toshiba Corp ${ }^{[[14]]}$ & All fingers & Kinematics signals & Impedance control strategy \\
\hline $\begin{array}{c}\text { National University of Singapore } \\
I^{[[15]]}\end{array}$ & Thumb & EMG signal & EMG-based “Low-level” control strategy \\
\hline MRC-Glove $[[16]]$ & Thumb & EMG signal & EMG-based "Low-level” control strategy \\
\hline $\begin{array}{c}\text { National University of Singapore } \\
\text { II[171]] }\end{array}$ & $\begin{array}{l}\text { Thumb, middle finger, ring finger, } \\
\text { little finger }\end{array}$ & EMG signal & EMG-based "Low-level” control strategy \\
\hline Harvard University I [18] ${ }^{[3]]}$ & All fingers & EMG signal & EMG-based “Low-level” control strategy \\
\hline Harvard University II ${ }^{[[19]]}$ & All fingers & EMG signal & EMG-based "Low-level” control strategy \\
\hline
\end{tabular}

\section{CONCLUSION}

Soft robotic hand exoskeleton technology are still new than the rigid robotic hand exoskeletons. This paper reviewed the existing research results on soft robotic hand exoskeleton in terms of mechanical design and control system. The challenges surrounding soft robotic hand exoskeleton technologies such as the definition of numbers of DOFs and actuators, the choice of actuators, the design of flexible deformation segments, and the implementation of high-level control strategies for a robotic hand exoskeleton system. Though the soft hand exoskeleton is a novel technology and needed to development in further, we can see its application prospect in rehabilitation and assisting of patients.

\section{REFERENCES}

[1] Y. W. Hong, et al., "Lower extremity exoskeleton: review and challenges surrounding the technology and its role in rehabilitation of lower limbs," Australian Journal of Basic and Applied Sciences, vol. 7, p. 4, 2013.

[2] A. M. Dollar and H. Herr, "Lower extremity exoskeletons and active orthoses: Challenges and state-of-the-art," Ieee Transactions on Robotics, vol. 24, pp. 144-158, Feb 2008.

[3] P. Polygerinos, et al., "Soft Robotic Glove for Hand Rehabilitation and Task Specific Training," 2015 Ieee International Conference on Robotics and Automation (Icra), pp. 2913-2919, 2015.

[4] C. D. Takahashi, et al., "Robot-based hand motor therapy after stroke," Brain, vol. 131, pp. 425-37, Feb 2008.

[5] P. Maciejasz, et al., "A survey on robotic devices for upper limb rehabilitation," Journal of Neuroengineering and Rehabilitation, vol. 11, Jan 92014.

[6] M. M. Foumashi, et al., "State-of-the-art of hand exoskeleton systems," Rapp. tech. Università di Bologna-DIEM, 2011.

[7] M. D. Baker, et al., "Orthotic Hand-Assistive Exoskeleton," 2011 Ieee 37th Annual Northeast Bioengineering Conference (Nebec), 2011.
[8] U. Jeong, et al., "Implementation of various control algorithms for hand rehabilitation exercise using wearable robotic hand," Intelligent Service Robotics, vol. 6, pp. 181-189, Oct 2013.

[9] M. Nilsson, et al., "The Soft Extra Muscle System for Improving the Grasping Capability in Neurological Rehabilitation," 2012 Ieee Embs Conference on Biomedical Engineering and Sciences (Iecbes), 2012.

[10] M. A. Delph, 2nd, et al., "A soft robotic exomusculature glove with integrated sEMG sensing for hand rehabilitation," IEEE Int Conf Rehabil Robot, vol. 2013, p. 6650426, Jun 2013.

[11] S. Biggar and W. Yao, "Design and Evaluation of a Soft and Wearable Robotic Glove for Hand Rehabilitation," Ieee Transactions on Neural Systems and Rehabilitation Engineering, vol. 24, pp. 1071-1080, Oct 2016.

[12] T. Noritsugu, et al., "Wearable power assist device for hand grasping using pneumatic artificial rubber muscle," SICE 2004 Annual Conference, Vols 1-3, pp. 420-425, 2004.

[13] Y. Kadowaki, et al., "Development of soft power-assist glove and control based on human intent," Journal of Robotics and Mechatronics, vol. 23(2), 2011.

[14] K. Toya, et al., "Power-assist glove operated by predicting the grasping mode," Journal of System Design and Dynamics, vol. 5, pp. 94-108, 2011.

[15] H. K. Yap, et al., "Design and Characterization of Soft Actuator for Hand Rehabilitation Application," 6th European Conference of the International Federation for Medical and Biological Engineering, vol. 45, pp. 367-370, 2015.

[16] H. K. Yap, et al., "MRC-Glove: A fMRI Compatible Soft Robotic Glove for Hand Rehabilitation Application," Proceedings of the Ieee/Ras-Embs International Conference on Rehabilitation Robotics (Icorr 2015), pp. 735-740, 2015.

[17] J. H. Low, et al., "Customizable Soft Pneumatic Finger Actuators for Hand Orthotic and Prosthetic Applications," Proceedings of the Ieee/Ras-Embs International Conference on Rehabilitation Robotics (Icorr 2015), pp. 380-385, 2015.

[18] P. Polygerinos, et al., "EMG Controlled Soft Robotic Glove for Assistance During Activities of Daily Living," Proceedings of the Ieee/Ras-Embs International Conference on Rehabilitation Robotics (Icorr 2015), pp. 55-60, 2015. 
[19] P. Polygerinos, et al., "Soft robotic glove for combined assistance and athome rehabilitation," Robotics and Autonomous Systems, vol. 73, pp. 135-143, Nov 2015.

[20] U. Onen, et al., "Design and Actuator Selection of a Lower Extremity Exoskeleton," Ieee-Asme Transactions on Mechatronics, vol. 19, pp. 623-632, Apr 2014.

[21] B. Buchholz and T. J. Armstrong, "A kinematic model of the human hand to evaluate its prehensile capabilities," J Biomech, vol. 25, pp. 14962, Feb 1992. 